\title{
Rational approximations to $\pi$ and some other numbers
}

\author{
by \\ Masayoshi Hata (Kyoto)
}

1. Introduction. In $1953 \mathrm{~K}$. Mahler [12] gave a lower bound for rational approximations to $\pi$ by showing that

$$
\left|\pi-\frac{p}{q}\right| \geq q^{-42}
$$

for any integers $p, q$ with $q \geq 2$. He also indicated that the exponent 42 can be replaced by 30 when $q$ is greater than some integer $q_{0}$. This result is based on the classical approximation formulae to the exponential and logarithmic functions due to Hermite. Our aim is to improve the knowledge of approximations by rational numbers of the classical constants of analysis such as $\pi$ and $\pi / \sqrt{3}$. In general, we say that a real number $\gamma$ has an irrationality measure $\mu \geq 2$ provided that, for any $\varepsilon>0$, there exists a positive integer $q_{0}(\varepsilon)$ satisfying

$$
\left|\gamma-\frac{p}{q}\right| \geq q^{-\mu-\varepsilon}
$$

for any integers $p, q$ with $q \geq q_{0}(\varepsilon)$.

Later the exponent 30 mentioned above was improved to 20 by M. Mignotte [13] by a refinement of Mahler's method, and to $19.8899944 \ldots$ by G. V. Chudnovsky [3]. Indeed, Chudnovsky has succeeded in determining the exact asymptotic behaviour of the complex integral

$$
\frac{1}{2 \pi i} \int_{C}\left(\frac{n !}{z(z-1) \ldots(z-n)}\right)^{k} e^{w z} d z
$$

as $n$ tends to infinity. Recently the author [11], modifying the above integral (1.1), obtained a fairly good irrationality measure 13.394 for $\pi$.

On the other hand, F. Beukers [2] gave an elegant irrationality proof of $\zeta(2)=\pi^{2} / 6$ by considering the double (real) integral 


$$
\int_{0}^{1} \int_{0}^{1} \frac{L(x)(1-y)^{n}}{1-x y} d x d y,
$$

where $L(x)=\frac{1}{n !}\left(x^{n}(1-x)^{n}\right)^{(n)}$ is the Legendre polynomial. The integral (1.2) gives an irrationality measure $11.85078 \ldots$ for $\pi^{2}$ and this has been pared down to 10.02979 by R. Dvornicich and C. Viola [9], to 7.552 by E. A. Rukhadze [15], to 7.5252 by the author [10], and to $7.51 \ldots$ by D. V. Chudnovsky and G. V. Chudnovsky [6]. (The measures 7.552 and 7.51 ... were announced without proofs.) Thus the last result due to Chudnovsky and Chudnovsky brings an irrationality measure $15.02 \ldots$ for $\pi$.

All the measures mentioned above are based on rational approximations to $\pi$ and its powers, though a single system of approximation to $\pi$ is desired. In this paper we consider the complex integral

$$
\int_{\Gamma}\left(F\left(a_{1}, a_{2}, a_{3} ; z\right)\right)^{n} \frac{d z}{z}
$$

where

$$
F\left(a_{1}, a_{2}, a_{3} ; z\right)=\frac{\left(z-a_{1}\right)^{2}\left(z-a_{2}\right)^{2}\left(z-a_{3}\right)^{2}}{z^{3}}
$$

with non-zero distinct complex numbers $a_{1}, a_{2}$ and $a_{3}$. By taking $a_{1}=1$, $a_{2}=2$ and $a_{3}=1+i$, the integral (1.3) enables us to obtain the following

TheOREM 1.1. For any $\varepsilon>0$, there exists a positive integer $H_{0}(\varepsilon)$ such that

$$
|p+q \pi+r \log 2| \geq H^{-\mu-\varepsilon}
$$

for any integers $p, q, r$ with $H \equiv \max \{|q|,|r|\} \geq H_{0}(\varepsilon)$, where the exponent $\mu$ is given by

$$
\mu=-\frac{2 \log \alpha_{0}+6-\pi / \sqrt{3}}{2 \log \alpha_{1}+6-\pi / \sqrt{3}}
$$

with

$\alpha_{j}=\frac{10}{9} \sqrt{18265} \cos \left(\theta_{0}+\frac{4 j \pi}{3}\right)+\frac{92}{3} \sqrt{6} \quad$ and $\quad \theta_{0}=\frac{1}{3} \arctan \left(\frac{23 \sqrt{69}}{1209303}\right)$. (Numerically one has $\mu=7.016045 \ldots$ )

In particular, we have

$$
\left|\pi-\frac{p}{q}\right| \geq q^{-8.0161} \quad \text { and } \quad\left|\frac{\pi}{\log 2}-\frac{p}{q}\right| \geq q^{-8.0161}
$$

for any integers $p, q$ with $q \geq q_{0}$. Hence the former remarkably improves the earlier irrationality measures of $\pi$ mentioned above.

As another application of the integral (1.3), by taking $a_{1}=1, a_{2}=$ $(1+\sqrt{3} i) / 2$ and $a_{3}=(3+\sqrt{3} i) / 4$, we have the following 
TheOREM 1.2. For any $\varepsilon>0$, there exists a positive integer $q_{0}(\varepsilon)$ such that

$$
\left|\frac{\pi}{\sqrt{3}}-\frac{p}{q}\right| \geq q^{-\xi-\varepsilon}
$$

for any integers $p, q$ with $q \geq q_{0}(\varepsilon)$, where the exponent $\xi$ is given by

$$
\xi=1+\frac{2 \log (\beta / 54)+6-\pi / \sqrt{3}}{\log (\beta / 3)-6+\pi / \sqrt{3}}
$$

with $\beta=4529+\sqrt[3]{u+v}+\sqrt[3]{u-v}, u=92868417494$ and $v=319599 \sqrt{5073}$. (Numerically one has $\xi=4.601579 \ldots$ )

Concerning the number $\pi / \sqrt{3}, \mathrm{~K}$. Alladi and M. L. Robinson [1] obtained an irrationality measure 8.30998 ... by using the Legendre polynomials. Later this was improved to 5.7926 by G. V. Chudnovsky [4], to 5.516 by A. K. Dubitskas [8], to $5.0874 \ldots$ by the author [10], and to 4.97 by G. Rhin [14]. (The last measure 4.97 was announced without proof.) Therefore our Theorem 1.2 improves all the measures for $\pi / \sqrt{3}$ mentioned above.

The principal part of the integral (1.3), as $n$ tends to $+\infty$, can be easily obtained by the saddle method originated in Riemann's work, which is described in detail in Dieudonné's book [7]. This may be regarded as a natural complex version of the well-known formula

$$
\lim _{n \rightarrow \infty}\left(\int_{0}^{1} \varphi(t)(f(t))^{n} d t\right)^{1 / n}=\max _{0 \leq t \leq 1} f(t)
$$

for real-valued positive continuous functions $\varphi(t)$ and $f(t)$. Owing to this method we do not need in this paper any discussion on recurrence relations of polynomials. We note that our new exponents appearing in Theorems 1.1 and 1.2 contain the roots of some cubic equations with integral coefficients.

We prepare four lemmas in the next section. In particular, Lemma 2.2 is an elementary arithmetical lemma concerning binomial coefficients, which plays an important role in this paper. Then Theorems 1.1 and 1.2 will be proved in Sections 3 and 4 respectively.

2. Preliminaries. First of all we need the following lemma concerning linear independences over $\mathbb{Q}$ :

Lemma 2.1. Let $m$ be a fixed non-negative integer. Let $\gamma_{1}$ and $\gamma_{2}$ be real numbers satisfying

$$
q_{n} \gamma_{1}-p_{n}=\varepsilon_{n} \quad \text { and } \quad q_{n} \gamma_{2}-r_{n}=\delta_{n}
$$

for some $p_{n}, q_{n}, r_{n} \in \mathbb{Z}+i \sqrt{m} \mathbb{Z}$ for all $n \geq 1$. Suppose that

$$
\lim _{n \rightarrow \infty} \frac{1}{n} \log \left|q_{n}\right|=\sigma, \quad \lim _{n \rightarrow \infty} \frac{1}{n} \log \left|\varepsilon_{n}\right|=-\tau, \quad \lim _{n \rightarrow \infty} \frac{1}{n} \log \left|\delta_{n}\right|=-\tau^{\prime}
$$


for positive numbers $\sigma, \tau$ and $\tau^{\prime}$ with $\tau^{\prime} \geq \tau$. Suppose further that there exist infinitely many $n$ 's satisfying $\delta_{n} / \varepsilon_{n} \neq \varrho$ for any rational number $\varrho$. Then the numbers $1, \gamma_{1}$ and $\gamma_{2}$ are linearly independent over $\mathbb{Q}$. More precisely, for any $\varepsilon>0$, there exists a positive integer $H_{0}(\varepsilon)$ such that

$$
\left|p+q \gamma_{1}+r \gamma_{2}\right| \geq H^{-\sigma / \tau-\varepsilon}
$$

for any integers $p, q, r$ with $H \equiv \max \{|q|,|r|\} \geq H_{0}(\varepsilon)$.

Proof. Obviously there exists an integer $n_{0}$ such that $q_{n} \neq 0, \varepsilon_{n} \neq 0$ and $\delta_{n} \neq 0$ for all $n \geq n_{0}$. Putting $\Lambda=p+q \gamma_{1}+r \gamma_{2}$ for arbitrarily fixed integers $(p, q, r) \neq(0,0,0)$, we have

$$
\begin{aligned}
\Lambda & =p+q\left(\frac{p_{n}+\varepsilon_{n}}{q_{n}}\right)+r\left(\frac{r_{n}+\delta_{n}}{q_{n}}\right) \\
& =\frac{p q_{n}+q p_{n}+r r_{n}}{q_{n}}+\frac{q \varepsilon_{n}+r \delta_{n}}{q_{n}} \equiv \frac{A_{n}}{q_{n}}+\frac{\omega_{n}}{q_{n}}, \quad \text { say. }
\end{aligned}
$$

We first show that the set $\Omega=\left\{n \geq 1: A_{n} \neq 0\right\}$ is infinite. Suppose, on the contrary, that $\Omega$ is finite; that is, $A_{n}=0$ for all $n \geq n_{1}$ for some integer $n_{1} \geq n_{0}$. Since $\left|q_{n}\right| \geq 1$ and $\omega_{n} \rightarrow 0$ as $n \rightarrow \infty$, we have $\Lambda=0$. Hence $\omega_{n}=0 ;$ so,

$$
q+r \frac{\delta_{n}}{\varepsilon_{n}}=0
$$

for all $n \geq n_{1}$. Since $(q, r) \neq(0,0)$, we have $r \neq 0$; hence $\delta_{n} / \varepsilon_{n}=-q / r$ for all $n \geq n_{1}$, contrary to the hypothesis of the lemma.

For any $\varepsilon>0$, one can define a sufficiently small $\delta \equiv \delta(\varepsilon) \in(0, \tau / 6)$ satisfying

$$
\frac{\sigma+\delta}{\tau-3 \delta}<\frac{\sigma}{\tau}+\frac{\varepsilon}{2}
$$

Then there exists an integer $n(\varepsilon) \geq n_{0}$ such that

$$
e^{(\sigma-\delta) n} \leq\left|q_{n}\right| \leq e^{(\sigma+\delta) n} \quad \text { and } \quad \max \left\{\left|\varepsilon_{n}\right|,\left|\delta_{n}\right|\right\} \leq e^{-(\tau-\delta) n}
$$

for all $n \geq n(\varepsilon)$. We now define

$$
H_{0}(\varepsilon)=\min \left\{N \geq 1: 4 N \geq e^{(\tau-\delta) n(\varepsilon)} \text { and } N^{\varepsilon \tau} \geq\left(4 e^{\sigma+\tau}\right)^{2 \tau+4 \sigma}\right\}
$$

and

$$
N(H)=\min \left\{N>n(\varepsilon): 4 H<e^{(\tau-\delta) N}\right\}
$$

for all $H \geq H_{0}(\varepsilon)$. Since $\left|\omega_{n}\right| \leq 2 H e^{-(\tau-\delta) n}$ for all $n \geq n(\varepsilon)$, we have $\left|\omega_{n}\right|<1 / 2$ for all $n \geq N(H)$. Therefore

$$
|\Lambda|=\left|\frac{A_{n}+\omega_{n}}{q_{n}}\right| \geq \frac{1-\left|\omega_{n}\right|}{\left|q_{n}\right|}>\frac{1}{2\left|q_{n}\right|} \geq \frac{1}{2} e^{-(\sigma+\delta) n}
$$

for any $n \in \Omega$ with $n \geq N(H)$. (Note that $\left|A_{n}\right| \geq 1$ if $A_{n} \neq 0$, since $A_{n} \in \mathbb{Z}+i \sqrt{m} \mathbb{Z}$.) 
Now we distinguish two cases as follows: (a) $N(H) \in \Omega$ or (b) $N(H) \notin \Omega$. In Case (a), it follows from the definition of $N(H)$ that $4 H \geq e^{(\tau-\delta)(N(H)-1)}$; so,

$$
e^{N(H)} \leq 2^{3 / \tau} e H^{1 /(\tau-\delta)} .
$$

Hence, putting $n=N(H)$ in $(2.1)$, we have

$$
|\Lambda| \geq \frac{1}{2} e^{-(\sigma+\delta) N(H)} \geq\left(H_{0}(\varepsilon)\right)^{-\varepsilon / 2} H^{-\sigma / \tau-\varepsilon / 2} \geq H^{-\sigma / \tau-\varepsilon},
$$

as required.

In Case (b), let $M(H)$ be the smallest integer satisfying $M(H)>N(H)$ and $M(H) \in \Omega$. Since $A_{M(H)-1}=0$, we have

$$
|\Lambda|=\left|\frac{\omega_{M(H)-1}}{q_{M(H)-1}}\right| \leq 2 H e^{-(\sigma+\tau-2 \delta)(M(H)-1)} ;
$$

so,

$$
e^{M(H)} \leq e\left(\frac{2 H}{|\Lambda|}\right)^{1 /(\sigma+\tau-2 \delta)} .
$$

Hence, putting $n=M(H)$ in (2.1), we obtain

$$
|\Lambda| \geq \frac{1}{2} e^{-(\sigma+\delta) M(H)} \geq \frac{1}{2} e^{-(\sigma+\delta)}\left(\frac{|\Lambda|}{2 H}\right)^{(\sigma+\delta) /(\sigma+\tau-2 \delta)} ;
$$

thus,

$$
\begin{aligned}
|\Lambda| & \geq\left(4 e^{\sigma+\tau}\right)^{-1-2 \sigma / \tau} H^{-(\sigma+\delta) /(\tau-3 \delta)} \\
& \geq\left(H_{0}(\varepsilon)\right)^{-\varepsilon / 2} H^{-\sigma / \tau-\varepsilon / 2} \geq H^{-\sigma / \tau-\varepsilon} .
\end{aligned}
$$

This completes the proof.

Remark 2.1. By the same argument as above, one can show that if $q_{n} \gamma-p_{n}=\varepsilon_{n}$ for some irrational number $\gamma$,

$$
\lim _{n \rightarrow \infty} \frac{1}{n} \log \left|q_{n}\right|=\sigma \quad \text { and } \quad \limsup _{n \rightarrow \infty} \frac{1}{n} \log \left|\varepsilon_{n}\right| \leq-\tau,
$$

then $\gamma$ has an irrationality measure $1+\sigma / \tau$. This slightly improves Proposition 3.3 in [10], in which the author posed the unnecessary hypothesis that $\gamma$ is not a Liouville number. This remark will be used in the proof of Theorem 1.2.

We next give an elementary arithmetical lemma concerning binomial coefficients.

Lemma 2.2. There exists a positive integer $D_{n}$ such that

$$
\frac{D_{n}}{j+k+l-3 n}\left(\begin{array}{c}
2 n \\
j
\end{array}\right)\left(\begin{array}{c}
2 n \\
k
\end{array}\right)\left(\begin{array}{c}
2 n \\
l
\end{array}\right) \in \mathbb{Z}
$$


for all integers $0 \leq j, k, l \leq 2 n$ with $j+k+l \neq 3 n$ and that

$$
\lim _{n \rightarrow \infty} \frac{1}{n} \log D_{n}=\frac{1}{2}\left\{6-\frac{\pi}{\sqrt{3}}+\log \frac{27}{16}\right\} \equiv \kappa, \quad \text { say. }
$$

(Numerically one has $\kappa=2.35472 \ldots$ )

Proof. Let $v(s, p)$ be the exponent of any prime factor $p$ in the resolution of each integer $1 \leq s \leq 3 n$ into its prime factors; that is,

$$
s=\prod_{p \text { prime }} p^{v(s, p)} .
$$

Then clearly

$$
v(s, p) \leq\left[\frac{\log s}{\log p}\right] \leq\left[\frac{\log (3 n)}{\log p}\right] .
$$

We next show that any prime number $p>\sqrt{3 n}$ belonging to the set

$$
T_{n}=\left\{p \text { prime }: \frac{1}{2} \leq\left\{\frac{n}{p}\right\}<\frac{2}{3}\right\},
$$

divides all the integers

$$
\left(\begin{array}{c}
2 n \\
j
\end{array}\right)\left(\begin{array}{c}
2 n \\
k
\end{array}\right)\left(\begin{array}{c}
2 n \\
l
\end{array}\right)
$$

where $(j, k, l)$ runs through the set $\Xi(n, p)=\{(j, k, l): 0 \leq j, k, l \leq 2 n$ and $j+k+l \equiv 3 n(\bmod p)\} .(\{x\}$ denotes the fractional part of $x$.$) To see this,$ it suffices to show that

$$
\begin{aligned}
J=\left[\frac{2 n}{p}\right]-\left[\frac{j}{p}\right]-\left[\frac{2 n-j}{p}\right]+\left[\frac{2 n}{p}\right] & -\left[\frac{k}{p}\right]-\left[\frac{2 n-k}{p}\right] \\
& +\left[\frac{2 n}{p}\right]-\left[\frac{l}{p}\right]-\left[\frac{2 n-l}{p}\right] \geq 1
\end{aligned}
$$

for any $(j, k, l) \in \Xi(n, p)$, since $p>\sqrt{3 n}$. Let $\omega=\{n / p\}, \theta=\{j / p\}$, $\theta^{\prime}=\{k / p\}$ and $\theta^{\prime \prime}=\{l / p\}$. Then obviously

$$
\begin{aligned}
J & =[2 \omega]-[2 \omega-\theta]+[2 \omega]-\left[2 \omega-\theta^{\prime}\right]+[2 \omega]-\left[2 \omega-\theta^{\prime \prime}\right] \\
& =3-[2 \omega-\theta]-\left[2 \omega-\theta^{\prime}\right]-\left[2 \omega-\theta^{\prime \prime}\right]
\end{aligned}
$$

since $[2 \omega]=1$. On the other hand, since $3 n=j+k+l+p q$ for some integer $q$ and since $[3 \omega]=1$, we have

$$
\{3 \omega\}=\left\{\theta+\theta^{\prime}+\theta^{\prime \prime}\right\}=3 \omega-1 .
$$

Thus $\theta+\theta^{\prime}+\theta^{\prime \prime}=3 \omega-1+r$ for some integer $r \geq 0$; hence

$$
\begin{aligned}
{[2 \omega-\theta]+\left[2 \omega-\theta^{\prime}\right]+\left[2 \omega-\theta^{\prime \prime}\right] } & \leq\left[6 \omega-\theta-\theta^{\prime}-\theta^{\prime \prime}\right]=[6 \omega-3 \omega+1-r] \\
& =1-r+[3 \omega]=2-r \leq 2 .
\end{aligned}
$$

Therefore we have $J \geq 1$, as required. 
We define

$$
\Delta_{1}(n)=\prod_{\substack{p \text { prime } \\ p \leq \sqrt{3 n}}} p^{\left[\frac{\log (3 n)}{\log p}\right]}, \quad \Delta_{2}(n)=\prod_{\substack{p \text { prime } \\ p \leq 3 n}} p, \quad \Delta_{3}(n)=\prod_{\substack{p \text { prime } \\ p \in T_{n}}} p
$$

for all $n \geq 1$. The above argument implies that the integer

$$
D_{n}=\frac{\Delta_{1}(n) \Delta_{2}(n)}{\Delta_{3}(n)}
$$

satisfies (2.2) for any integers $j, k, l \in[0,2 n]$ with $j+k+l \neq 3 n$. Put $b_{i}=3 /(3 i+2), c_{i}=2 /(2 i+1)$ and

$$
S_{i}(n)=\frac{1}{n} \sum_{\substack{p \text { prime } \\ p / n \in\left(b_{i}, c_{i}\right]}} \log p
$$

for $i \geq 0$ for brevity. Then clearly

$$
\frac{1}{n} \log \Delta_{3}(n)=\sum_{i=0}^{\infty} S_{i}(n)
$$

and it follows from the prime number theorem that $S_{i}(n) \rightarrow c_{i}-b_{i}$ as $n \rightarrow \infty$ for each $i \geq 0$.

Thus

$$
\begin{aligned}
\lim _{n \rightarrow \infty} \frac{1}{n} \log \Delta_{3}(n) & =\sum_{i=0}^{\infty} \lim _{n \rightarrow \infty} S_{i}(n)=\sum_{i=0}^{\infty}\left(c_{i}-b_{i}\right) \\
& =\frac{\Gamma^{\prime}(2 / 3)}{\Gamma(2 / 3)}-\frac{\Gamma^{\prime}(1 / 2)}{\Gamma(1 / 2)}=\frac{1}{2}\left\{\frac{\pi}{\sqrt{3}}-\log \frac{27}{16}\right\}
\end{aligned}
$$

where $\Gamma(z)$ is the gamma function. (The interchange of the operations of limit and summation can be easily justified by using the estimate

$$
\sum_{i \geq L} S_{i}(n) \leq \operatorname{const} c_{L}
$$

for any $L \geq 1$, where const depends neither on $L$ nor on $n$.) Since it can be seen that $\log \Delta_{1}(n)=O(\sqrt{n})$ and $\log \Delta_{2}(n) \sim 3 n$ as $n$ tends to $+\infty$, we thus obtain the asymptotic behaviour (2.3). This completes the proof.

We now consider the function $F(z) \equiv F\left(a_{1}, a_{2}, a_{3} ; z\right)$ defined in (1.4) for non-zero distinct complex numbers $a_{1}, a_{2}$ and $a_{3}$. It is easily seen that $F^{\prime}(z)$ has three zero points $\zeta_{1}, \zeta_{2}, \zeta_{3}$ in the region $\mathbb{C}-\left\{a_{1}, a_{2}, a_{3}\right\}$, which satisfy the cubic equation

$$
z^{3}+s_{2} z^{2}+s_{1} z+s_{0}=0
$$


with $s_{0}=a_{1} a_{2} a_{3}, s_{1}=-\left(a_{1} a_{2}+a_{2} a_{3}+a_{3} a_{1}\right) / 3$ and $s_{2}=-\left(a_{1}+a_{2}+a_{3}\right) / 3$. Such points $\zeta_{j}$ are called "saddles" of the surface $\Sigma$ of $|F(z)|$. Using the equalities

$$
F\left(\zeta_{j}\right)=4\left\{\left(s_{2}^{2}-3 s_{1}\right) \zeta_{j}+\frac{s_{1}^{2}-3 s_{0} s_{2}}{\zeta_{j}}-7 s_{1} s_{2}-s_{0}\right\}
$$

for $1 \leq j \leq 3$, one can easily calculate every elementary symmetric function on $F\left(\zeta_{1}\right), F\left(\zeta_{2}\right)$ and $F\left(\zeta_{3}\right)$. We thus have

Lemma 2.3. Put $t_{0}=s_{0}, t_{1}=s_{1} s_{2}$ and $t_{2}=s_{2}^{3}+s_{1}^{3} / s_{0}$ for brevity. Then we have the identity

$$
\prod_{j=1}^{3}\left(z-F\left(\zeta_{j}\right)\right)=z^{3}+4 \sigma_{2} z^{2}+16 \sigma_{1} z+64 \sigma_{0},
$$

where

$$
\begin{aligned}
\sigma_{0}= & t_{0}^{3}-12 t_{0}^{2} t_{1}-8 t_{0}^{2} t_{2}+30 t_{0} t_{1}^{2}+48 t_{0} t_{1} t_{2} \\
& +16 t_{0} t_{2}^{2}+36 t_{1}^{3}+24 t_{1}^{2} t_{2}+9 t_{1}^{4} / t_{0}, \\
\sigma_{1}= & 3 t_{0}^{2}+3 t_{0} t_{1}+20 t_{0} t_{2}+57 t_{1}^{2}+12 t_{1} t_{2}+t_{1}^{3} / t_{0}, \\
\sigma_{2}= & 3 t_{0}+15 t_{1}+t_{2} .
\end{aligned}
$$

Finally, we recall the following result from Dieudonné's book [7]:

LEMma 2.4. Let $\Gamma$ be a smooth oriented path with a finite length, which departs from $a_{j}$ and arrives at $a_{k}$ through a saddle $\zeta_{l}$ for some $1 \leq j, k, l \leq 3$. Put $\ell_{z}=\{z+t: t \geq 0\}$, the half-straight line parallel to the real axis. Suppose that the saddle $\zeta_{l}$ is a simple root of (2.4) and suppose further that $\Gamma-\left\{a_{j}, a_{k}\right\}$ is contained in the simply connected region $\mathbb{C}_{0} \equiv \mathbb{C}-$ $\left(\ell_{0} \cup \ell_{a_{1}} \cup \ell_{a_{2}} \cup \ell_{a_{3}}\right)$ and that $\Gamma-\left\{a_{j}, a_{k}, \zeta_{l}\right\}$ is contained in the open set $\left\{z \in \mathbb{C}_{0}:|F(z)|<\left|F\left(\zeta_{l}\right)\right|\right\}$. Then the principal part of the integral

$$
I_{n}=\int_{\Gamma}(F(z))^{n} \frac{d z}{z}
$$

as $n$ tends to $+\infty$, is given by

$$
I_{n} \sim d_{0} \frac{\left(F\left(\zeta_{l}\right)\right)^{n}}{\sqrt{n}} \quad \text { with } \quad d_{0}=\sqrt{-2 \pi \frac{F\left(\zeta_{l}\right)}{F^{\prime \prime}\left(\zeta_{l}\right)}} \cdot \frac{1}{\zeta_{l}}
$$

in particular, we have

$$
\lim _{n \rightarrow \infty} \frac{1}{n} \log \left|I_{n}\right|=\log \left|F\left(\zeta_{l}\right)\right| .
$$

Indeed, the above lemma is an immediate consequence of the result in 
[7; Chapter IX], since the integral $I_{n}$ can be expressed as

$$
I_{n}=(-1)^{n} \int_{\Gamma} g(z) e^{n h(z)} d z
$$

where $g(z)=1 / z$ and $h(z)=2 \sum_{j=1}^{3} \log \left(a_{j}-z\right)-3 \log (-z)$ are analytic functions in $\mathbb{C}_{0}$.

3. Proof of Theorem 1.1. Let $\Gamma_{z, w}$ be a smooth oriented path departing from $z$, arriving at $w$, and contained in $\mathbb{C}-\{0\}$. We then consider the integral

$$
\begin{aligned}
I_{n}\left(\Gamma_{z, w}\right) & \equiv \int_{\Gamma_{z, w}}\left(F\left(a_{1}, a_{2}, a_{3} ; z\right)\right)^{n} \frac{d z}{z} \\
& =\sum_{j=0}^{2 n} \sum_{k=0}^{2 n} \sum_{l=0}^{2 n} B_{j, k, l}\left(\begin{array}{c}
2 n \\
j
\end{array}\right)\left(\begin{array}{c}
2 n \\
k
\end{array}\right)\left(\begin{array}{c}
2 n \\
l
\end{array}\right) \int_{\Gamma_{z, w}} z^{j+k+l-3 n-1} d z,
\end{aligned}
$$

where

$$
B_{j, k, l}=(-1)^{j+k+l} a_{1}^{2 n-j} a_{2}^{2 n-k} a_{3}^{2 n-l} .
$$

Therefore we have the identity

$$
\begin{aligned}
& \sum_{j+k+l \neq 3 n} \frac{B_{n}\left(\Gamma_{z, w}\right)}{j+k+l-3 n}\left(\begin{array}{c}
2 n \\
j
\end{array}\right)\left(\begin{array}{c}
2 n \\
k
\end{array}\right)\left(\begin{array}{c}
2 n \\
l
\end{array}\right)\left(w^{j+k+l-3 n}-z^{j+k+l-3 n}\right) \\
& +\sum_{j+k+l=3 n} B_{j, k, l}\left(\begin{array}{c}
2 n \\
j
\end{array}\right)\left(\begin{array}{c}
2 n \\
k
\end{array}\right)\left(\begin{array}{c}
2 n \\
l
\end{array}\right) \int_{\Gamma_{z, w}} \frac{d z}{z} \\
\equiv & u_{n}(z, w)+v_{n} \int_{\Gamma_{z, w}} \frac{d z}{z}, \quad \text { say. }
\end{aligned}
$$

For the proof of Theorem 1.1 we choose $a_{1}=1, a_{2}=2$ and $a_{3}=1+i$. Then it follows from Lemma 2.3 that $w_{k} \equiv \frac{9}{4}(i-1) F\left(\zeta_{k}\right), 1 \leq k \leq 3$, satisfy the cubic equation

$$
9 w^{3}-4968 w^{2}+862 w-36=0 .
$$

This algebraic equation has three positive roots; indeed,

$$
w_{k}=\frac{10}{9} \sqrt{109590} \cos \left(\theta_{0}+\frac{2 k \pi}{3}\right)+184
$$

for $1 \leq k \leq 3$ where $\theta_{0}=\frac{1}{3} \arctan \left(\frac{23 \sqrt{69}}{1209303}\right)$. We thus have $\left|F\left(\zeta_{k}\right)\right|=$ $\frac{2}{9} \sqrt{2} w_{k}$. Let $\Gamma_{1,2}$ and $\Gamma_{1,1+i}$ be the paths illustrated in Figure 1 through 
the saddles $\zeta_{1}$ and $\zeta_{2}$ respectively. Then clearly

$$
\int_{\Gamma_{1,2}} \frac{d z}{z}=\log 2 \quad \text { and } \quad \int_{\Gamma_{1,1+i}} \frac{d z}{z}=\frac{1}{2} \log 2+\frac{\pi}{4} i .
$$

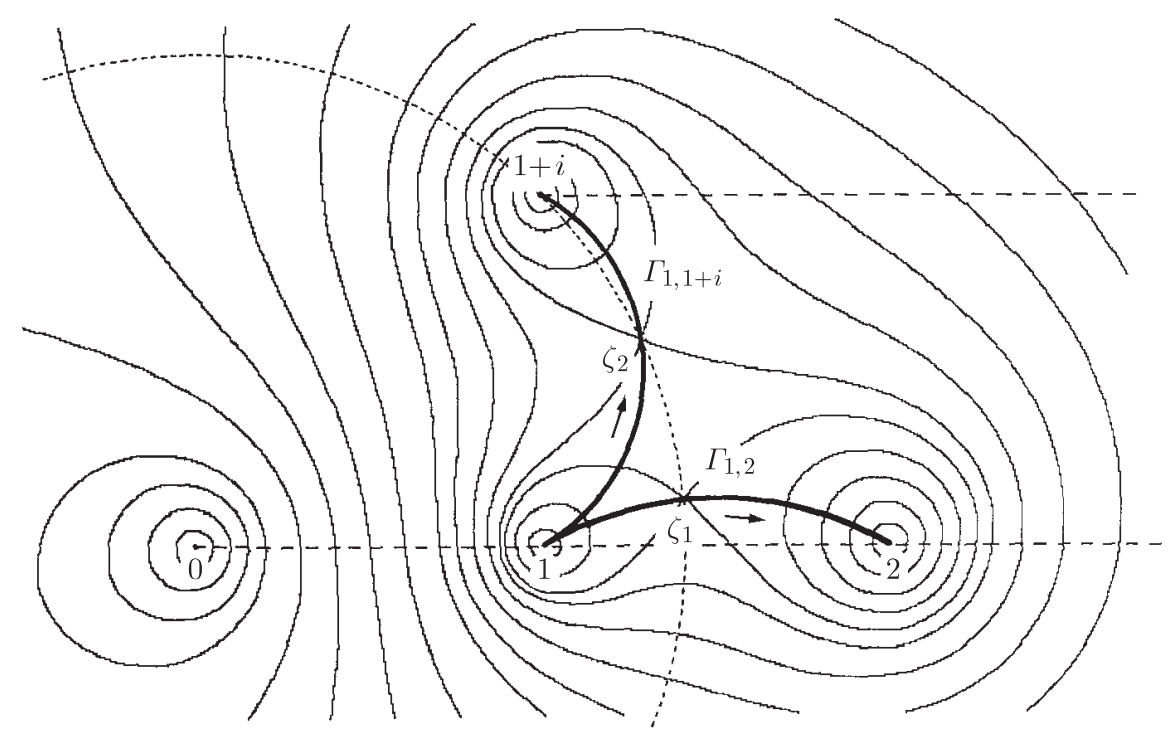

Fig. 1

Since

$$
(-1)^{j+k+l} B_{j, k, l} 2^{j+k+l-3 n}=2^{j+l-n}(1+i)^{2 n-l}=2^{j} i^{n}(1-i)^{l}
$$

and

$(-1)^{j+k+l} B_{j, k, l}(1+i)^{j+k+l-3 n}=2^{2 n-k}(1+i)^{j+k-n}=(1+i)^{j+n}(1-i)^{2 n-k}$,

it follows from Lemma 2.2 that both $u_{n}(1,2)$ and $u_{n}(1,1+i)$ belong to the set $(\mathbb{Z}+i \mathbb{Z}) / D_{n}$. Hence, putting

$p_{n}=-2 i D_{n}\left\{u_{n}(1,2)-2 u_{n}(1,1+i)\right\}, \quad q_{n}=D_{n} v_{n}, \quad r_{n}=-D_{n} u_{n}(1,2)$,

we obtain

$$
q_{n} \pi-p_{n}=2 i D_{n}\left\{I_{n}\left(\Gamma_{1,2}\right)-2 I_{n}\left(\Gamma_{1,1+i}\right)\right\} \equiv \varepsilon_{n}
$$

and

$$
q_{n} \log 2-r_{n}=D_{n} I_{n}\left(\Gamma_{1,2}\right) \equiv \delta_{n}, \quad \text { say, }
$$

where $p_{n}, q_{n}, r_{n} \in \mathbb{Z}+i \mathbb{Z}$. Then it follows from Lemmas 2.2 and 2.4 that

$$
\tau=-\lim _{n \rightarrow \infty} \frac{1}{n} \log \left|\varepsilon_{n}\right|=-\kappa-\log \left|F\left(\zeta_{2}\right)\right|
$$


and

$$
\tau^{\prime}=-\lim _{n \rightarrow \infty} \frac{1}{n} \log \left|\delta_{n}\right|=-\kappa-\log \left|F\left(\zeta_{1}\right)\right|,
$$

since $\left|F\left(\zeta_{1}\right)\right|<\left|F\left(\zeta_{2}\right)\right|$. Moreover, the hypothesis on the sequence $\left\{\delta_{n} / \varepsilon_{n}\right\}$ in Lemma 2.1 is clearly satisfied since $\tau^{\prime}>\tau$.

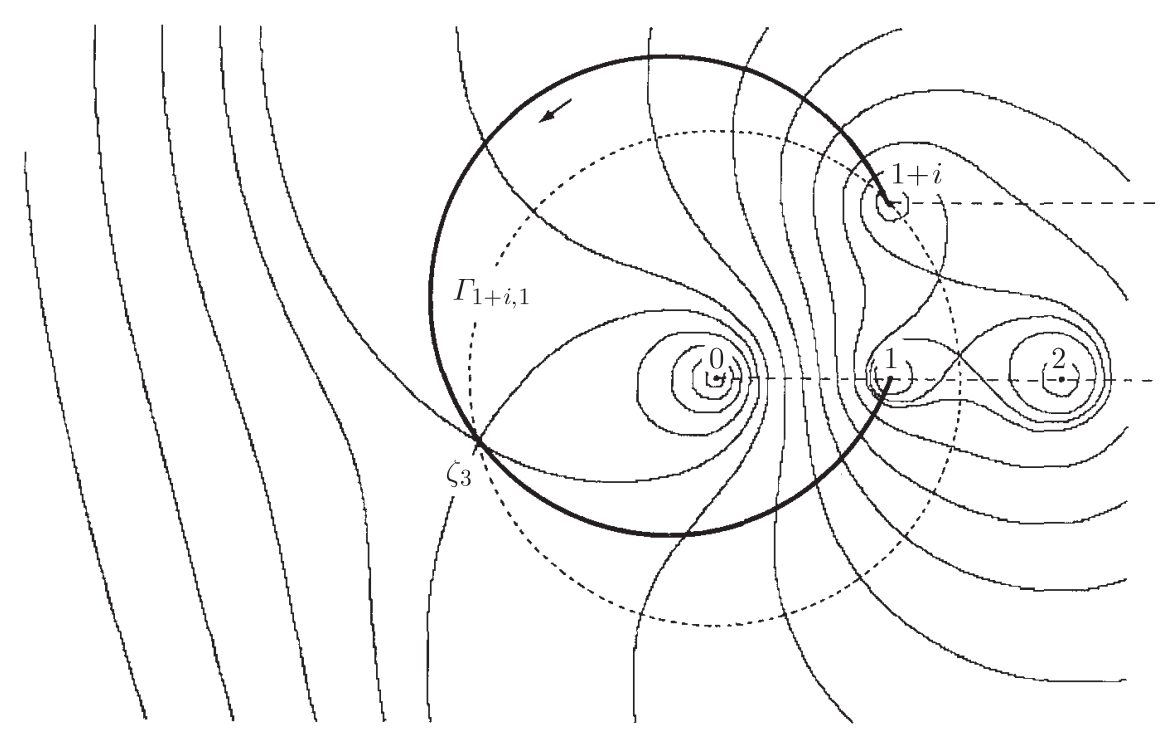

Fig. 2

On the other hand,

$$
v_{n}=\sum_{j+k+l=3 n} B_{j, k, l}\left(\begin{array}{c}
2 n \\
j
\end{array}\right)\left(\begin{array}{c}
2 n \\
k
\end{array}\right)\left(\begin{array}{c}
2 n \\
l
\end{array}\right)=\frac{1}{2 \pi i} \int_{C}(F(z))^{n} \frac{d z}{z},
$$

where $C=\Gamma_{1,1+i} \cup \Gamma_{1+i, 1}$ is a closed oriented curve enclosing the origin and $\Gamma_{1+i, 1}$ is the path illustrated in Figure 2 through the saddle $\zeta_{3}$. Hence

$$
\sigma=\lim _{n \rightarrow \infty} \frac{1}{n} \log \left|q_{n}\right|=\kappa+\log \left|F\left(\zeta_{3}\right)\right|
$$

since $\left|F\left(\zeta_{3}\right)\right|>\left|F\left(\zeta_{2}\right)\right|$. Therefore, by Lemma 2.1, the numbers $1, \log 2$ and $\pi$ have a linear independence measure

$$
\mu=\frac{\sigma}{\tau}=-\frac{\kappa+\log \left|F\left(\zeta_{3}\right)\right|}{\kappa+\log \left|F\left(\zeta_{2}\right)\right|}
$$

This completes the proof.

4. Proof of Theorem 1.2. We take $a_{1}=1, a_{2}=\lambda, a_{3}=\lambda^{\prime}=(1+\lambda) / 2$ with $\lambda=e^{\pi i / 3}$. Then it follows from Lemma 2.3 that $w_{k}=3 \sqrt{3} i F\left(\zeta_{k}\right)$, 
$1 \leq k \leq 3$, satisfy the cubic equation

$$
18 w^{3}-4529 w^{2}+81 w-1=0 .
$$

Indeed, this algebraic equation has one positive root

$$
w_{3}=\frac{1}{54}(4529+\sqrt[3]{u+v}+\sqrt[3]{u-v}) \equiv \frac{\beta}{54}, \quad \text { say },
$$

with $u=92868417494$ and $v=319599 \sqrt{5073}$, and two complex roots $w_{1}, w_{2}=\bar{w}_{1}$. We thus have

$$
\left|F\left(\zeta_{3}\right)\right|=\frac{\sqrt{3}}{9} w_{3}=\frac{\sqrt{3} \beta}{486} \quad \text { and } \quad\left|F\left(\zeta_{1}\right)\right|=\left|F\left(\zeta_{2}\right)\right|=\frac{1}{3 \sqrt{\beta}}
$$

since $w_{1} w_{2} w_{3}=1 / 18$. Let $\Gamma_{1, \lambda}$ be the path illustrated in Figure 3 through the saddles $\zeta_{1}$ and $\zeta_{2}$. Then clearly

$$
\int_{\Gamma_{1, \lambda}} \frac{d z}{z}=\log \lambda=\frac{\pi}{3} i
$$

and

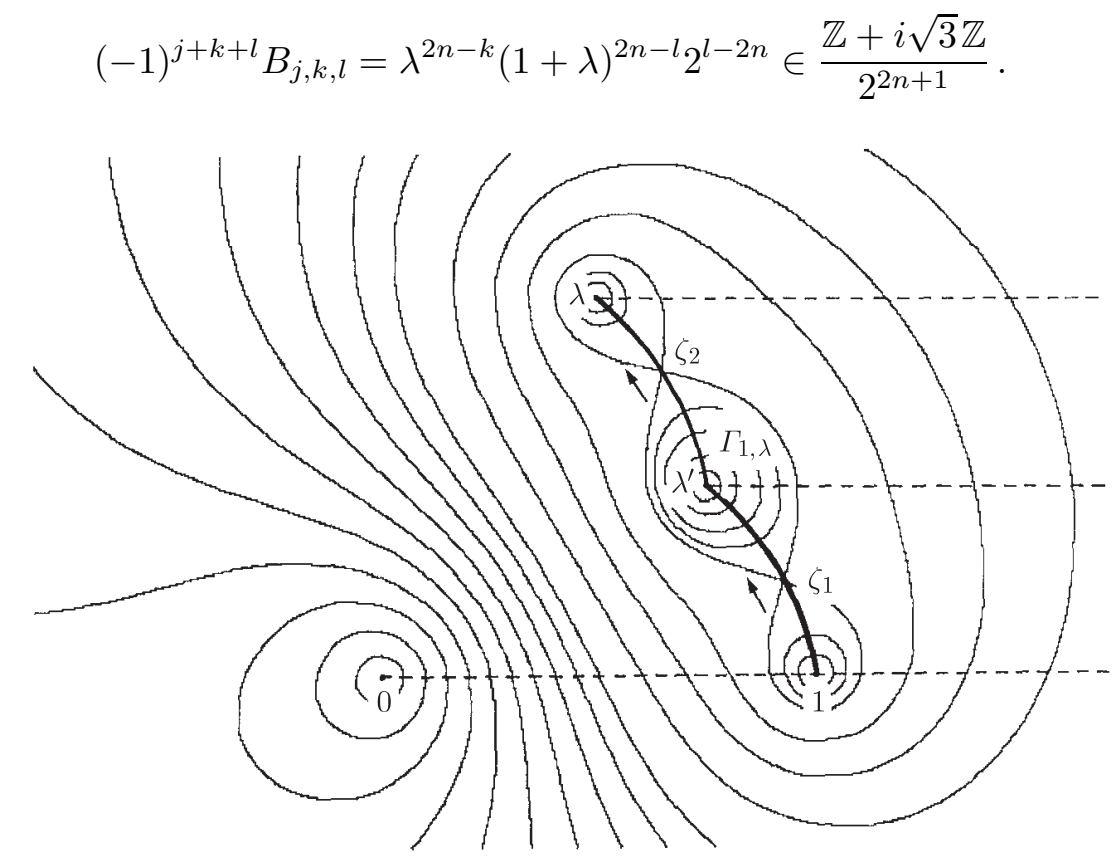

Fig. 3

Hence, putting

$$
q_{n}=2^{2 n+1} D_{n} v_{n} \quad \text { and } \quad p_{n}=2^{2 n+1} \sqrt{3} i D_{n} u_{n}(1, \lambda),
$$


we obtain, from (3.1),

$$
q_{n} \frac{\pi}{\sqrt{3}}-p_{n}=-2^{2 n+1} \sqrt{3} i D_{n} I_{n}\left(\Gamma_{1, \lambda}\right) \equiv \varepsilon_{n}, \quad \text { say, }
$$

where $p_{n}, q_{n} \in \mathbb{Z}+i \sqrt{3} \mathbb{Z}$. Then it follows from Lemmas 2.2 and 2.4 that

$$
\begin{aligned}
\limsup _{n \rightarrow \infty} \frac{1}{n} \log \left|\varepsilon_{n}\right| & \leq \log 4+\kappa+\limsup _{n \rightarrow \infty} \frac{1}{n} \log \left(\left|I_{n}\left(\Gamma_{1, \lambda^{\prime}}\right)\right|+\left|I_{n}\left(\Gamma_{\lambda^{\prime}, \lambda}\right)\right|\right) \\
& \leq \log 4+\kappa+\log \left|F\left(\zeta_{1}\right)\right| \equiv-\tau, \quad \text { say, }
\end{aligned}
$$

and that

$$
\sigma=\lim _{n \rightarrow \infty} \frac{1}{n} \log \left|q_{n}\right|=\log 4+\kappa+\log \left|F\left(\zeta_{3}\right)\right|
$$

since

$$
v_{n}=\frac{1}{2 \pi i} \int_{C}(F(z))^{n} \frac{d z}{z}
$$

where $C=\Gamma_{1, \lambda} \cup \Gamma_{\lambda, 1}$ is a closed curve enclosing the origin and $\Gamma_{\lambda, 1}$ is the path illustrated in Figure 4 through the saddle $\zeta_{3}$. Therefore, by Remark 2.1, the number $\pi / \sqrt{3}$ has an irrationality measure

$$
\xi=1+\frac{\sigma}{\tau}=1-\frac{\log 4+\kappa+\log \left|F\left(\zeta_{3}\right)\right|}{\log 4+\kappa+\log \left|F\left(\zeta_{1}\right)\right|} .
$$

This completes the proof of Theorem 1.2.

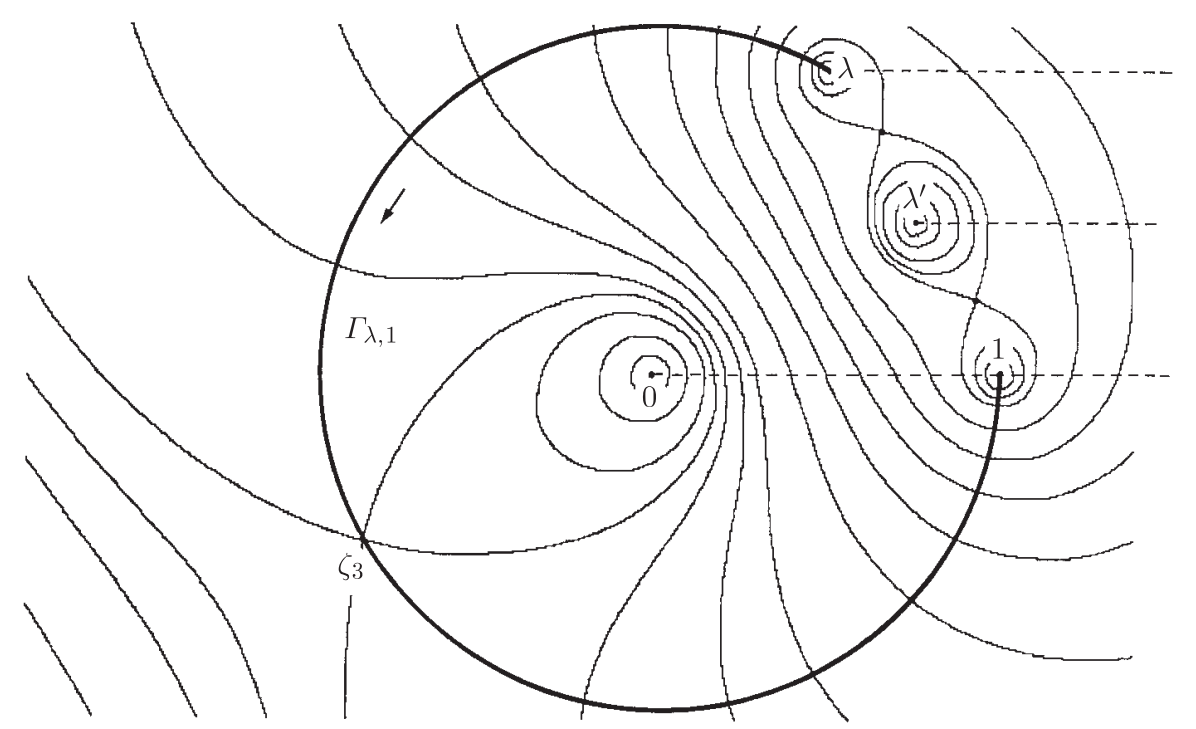

Fig. 4 
5. Remarks. Using Lemma 2.1 one can also obtain a linear independence measure for the numbers $1, \pi / \sqrt{3}$ and $\log (3 / 4)$ from the integral considered in Section 4. However, it can be seen that the case in which $a_{1}=3 / 2, a_{2}=2$ and $a_{3}=1+\lambda$ with $\lambda=e^{\pi i / 3}$ gives a better linear independence measure $\mu$ for such numbers. Indeed, we have

$$
\mu=-\frac{\log 8+\kappa+\log \left|x_{3}\right|}{\log 8+\kappa+\log \left|x_{2}\right|}=7.813765 \ldots,
$$

where $w_{k}=-(1 / \sqrt{3}) e^{-\pi i / 6} x_{k}, 1 \leq k \leq 3$, are three positive roots of the cubic equation $8748 w^{3}-1621134 w^{2}+3547 w-1=0$ satisfying $w_{1}<w_{2}<w_{3}$. The hypothesis on $\left\{\delta_{n} / \varepsilon_{n}\right\}$ in Lemma 2.1 is clearly satisfied since $\tau^{\prime}>\tau$. (We must multiply both sides of (3.1) by the factor $2^{3 n+1} D_{n}$.) In particular,

$$
\left|\frac{\pi}{\sqrt{3} \log (3 / 4)}-\frac{p}{q}\right| \geq q^{-8.8138}
$$

for any integer $p$ and for sufficiently large integer $q$.

Similarly one can obtain a linear independence measure $\mu^{\prime}$ for the numbers $1, \pi / \sqrt{3}$ and $\log 3$ by taking $a_{1}=1, a_{2}=\lambda$ and $a_{3}=1+\lambda$. In fact,

$$
\mu^{\prime}=-\frac{\frac{1}{2} \log 3+\kappa+\log \left|y_{3}\right|}{\frac{1}{2} \log 3+\kappa+\log \left|y_{1}\right|}=8.385245 \ldots,
$$

where $w_{k}=\frac{3}{4} \sqrt{3} i y_{k}, 1 \leq k \leq 3$, are three roots of the cubic equation $9 w^{3}-1187 w^{2}-61 w-1=0$ satisfying $w_{3}>0$ and $w_{1}=\bar{w}_{2}$. (We must multiply both sides of $(3.1)$ by the factor $2(1+\lambda)^{n} D_{n}$.) In this case we have $\left|y_{1}\right|=\left|y_{2}\right|$ (that is, $\tau^{\prime}=\tau$ ); so it follows from Lemma 2.4 that

$$
\frac{\delta_{n}}{\varepsilon_{n}}=d^{*} e^{i n \nu}+o(1)
$$

as $n$ tends to $+\infty$, where $d^{*} \neq 0$ is some complex constant and $\nu=\arg w_{1}-$ $\arg w_{2}$ satisfies $0<|\nu|<\pi$. Hence the sequence $\left\{\delta_{n} / \varepsilon_{n}\right\}$ satisfies the hypothesis of Lemma 2.1, as required. In particular,

$$
\left|\frac{\pi}{\sqrt{3} \log 3}-\frac{p}{q}\right| \geq q^{-9.3853}
$$

for any integer $p$ and for sufficiently large $q$.

\section{References}

[1] K. Alladi and M. L. Robinson, Legendre polynomials and irrationality, J. Reine Angew. Math. 318 (1980), 137-155.

[2] F. Beukers, A note on the irrationality of $\zeta(2)$ and $\zeta(3)$, Bull. London Math. Soc. 11 (1979), 268-272. 
[3] G. V. Chudnovsky, Hermite-Padé approximations to exponential functions and elementary estimates of the measure of irrationality of $\pi$, in: Lecture Notes in Math. 925, Springer, Berlin 1982, 299-322.

[4] —, Recurrences, Padé approximations and their applications, in: Lecture Notes in Pure and Appl. Math. 92, Dekker, New York 1984, 215-238.

[5] D. V. Chudnovsky and G. V. Chudnovsky, Padé and rational approximations to systems of functions and their arithmetic applications, in: Lecture Notes in Math. 1052, Springer, Berlin 1984, 37-84.

[6] - - - Transcendental methods and theta-functions, in: Proc. Sympos. Pure Math. 49 (2), Amer. Math. Soc., 1989, 167-232.

[7] J. Dieudonné, Calcul infinitésimal, Hermann, Paris 1968.

[8] A. K. Dubitskas, Approximation of $\pi / \sqrt{3}$ by rational fractions, Vestnik Moskov. Univ. Ser. I Mat. Mekh. 1987 (6), 73-76 (in Russian).

[9] R. Dvornicich and C. Viola, Some remarks on Beukers' integrals, in: Colloq. Math. Soc. János Bolyai 51, Budapest 1987, 637-657.

[10] M. Hata, Legendre type polynomials and irrationality measures, J. Reine Angew. Math. 407 (1990), 99-125.

[11] - A lower bound for rational approximations to $\pi$, J. Number Theory, to appear.

[12] K. Mahler, On the approximation of $\pi$, Nederl. Akad. Wetensch. Proc. Ser. A 56 (1953), 30-42.

[13] M. Mignotte, Approximations rationnelles de $\pi$ et quelques autres nombres, Bull. Soc. Math. France Mém. 37 (1974), 121-132.

[14] G. Rhin, Approximants de Padé et mesures effectives d'irrationalité, in: Progr. in Math. 71, Birkhäuser, 1987, 155-164.

[15] E. A. Rukhadze, A lower bound for the approximation of $\ln 2$ by rational numbers, Vestnik Moskov. Univ. Ser. I Mat. Mekh. 1987 (6), 25-29 (in Russian).

INSTITUTE OF MATHEMATICS

YOSHIDA COLLEGE

KYOTO UNIVERSITY

KYOTO 606, JAPAN

Received on 3.3.1992

and in revised form on 27.10 .1992 